cylinder. Acute rheumatism, so far as one knows, has not been thus treated. Sub-acute and chronic conditions of both gout and rheumatism are often either benefited or cured by the treatment. Last but not least, osteo-arthritis may be checked in its early stages, while later pain may be relieved, the movements of crippled joints restored, and the progress of the disease arrested. In making these general statements as to chronic joint stiffenings due regard must, of course, be paid to the condition of the joint. At the risk of some repetition it may be pointed out that in an advanced state of disorganisation all that can be hoped for is to relieve pain and check the progress of the disease.

CASE 3. Chronic monarticular rheumatism.-A man, aged 45 years, had chronic sub-acute pain of the right shoulder of about two years' duration. There was no clear family history of rheumatism or gout. The urine was normal. The pain was felt on certain movements of the joint. There was one acute attack in which the temperature ran up to $102^{\circ} \mathrm{F}$., with intense articular pain and tenderness. There were occasional twinges of pain in the right forearm and wrist. The patient used to ride a good deal, and sometimes got wet while hunting, but that did not increase the joint trouble. A glass or two of champagne, however, was almost certain to be followed by pain in the shoulder within 24 hours. Treatment of all kinds proved useless so far as a cure was concerned. Courses of drugs, salicylates, iodide of potassium, and alkalies were given. Diets of various kinds, including a vegetarian course, were tried. Prolonged massage and bath treatment at various spas-Bath, Droitwich, and Badenafforded nothing beyond temporary relief. The effect of the superheated-air bath was marked and immediate. After 11 baths the patient was almost free from pain. He had an apparatus at his own house, and after a few months he could strike out freely from the right shoulder. He was free for something like two years, when the rheumatism recurred in the left shoulder under conditions of bad living and exposure.

CASE 4.-The patient was a married woman, aged 50 years ; she had 10 children living. She came to the Free Institute on March 2nd, 1900, complaining of pain and swelling of the feet. Her father died, aged 46 years, from heart disease. Her eldest brother had "rheumatism." The patient had had rheumatic fever four times; the first attack was 22 years ago; she had had three attacks within three years. Six weeks previously swelling came on in the feet with great pain which had kept her awake at night and she had had to take sleeping draughts constantly. The hands were stiff and the arms could not be lifted above the level of the shoulders which crepitated and were tender on deep pressure. The eyes were puffed in the morning and she felt " tired." The heart's action was irregular, with loud mitral systolic and double aortic murmurs. She had suffered from cough for several winters. The specific gravity of the urine was 1020; there was no albumin. A diagnosis of chronic rheumatism with valvular disease of the heart with poor prognosis was made. Improvement under the superheated air was marked and rapid. After being treated four times the patient was able to walk better and to sleep at night without draughts. On May 30 th she could walk well for some distance. The movements of the hands were free and she could touch the top of her head with the left hand and almost with the right. No cardiac murmurs were heard on the last-mentioned date. Her general health was improved and she felt hungry on walking home after treatment.

The foresoing case shows that cardiac complications need not necessarily contra-indicate the application of the Tallerman treatment. It also shows the amount of good that can be effected even in most unpromising cases. A great number of further cases, illustrating the value of the treat ment, might be added. The few that have been noted will serve to indicate the chief directions of usefulness of this new remedy. Many more have been placed on record by good observers, both at home and abroad, as may be seen by a reference to Dr. Shadwell's monograph on the subject.

Bentinck-street, W.

WATER-SUPPLY OF NEWPORT (MON.). - The expenditure of the Newport Corporation on the Wentwood Water Works scheme up to the present amounts to £214,798.

SMall-POx Hospital for Barry.-At a meeting of the public health committee of the Barry District Council held on August 8th it was decided to erect a permanent small-pox hospital at an estimated cost of $£ 8160$.

\section{SOME REMARKS ON CANCER OF THE BREAST.}

BY SKENE KEITH, M.B., C.M., F.R.C.S. EdIN.

A VERY great deal of interest, perhaps even greater than has ever been the case before, appears at the present time to be taken in the disease cancer. The three questions usually asked by the general public are, Is it increasing? is it hereditary? and is it curable by operation? Concerning cancer of the breast I wish to bring forward some details, not new certainly, but which often do not seem to me to have had sufficient attention paid to them when the character of the operation in that region has been discussed. I make no apology for not entering into the first two questions, for it does not seem to me that we are in a position to answer either of them definitely. To answer the first, it would be necessary to have for comparison the statistics of two considerable periods of time, with-and this is of vital importance-an equal amount of knowledge on the part of those supplying the data for the statistics. Even if we were to attempt to compare the statistics of the past 10 years with those of the preceding decade we are faced with the fact that medical education has improved and more especially in the department of pathology. Although the microscope has done wonders to help us, it is not so many months ago since we had a case brought forward, by competent men, of a cure, temporary at least, of a sarcoma by the injection of Coley's fluid. The diagnosis bad been made not only clinically but also by the help of the microscope. Doubt was expressed as to the correctness of the diagnosis, and after a second microscopic examination of the specimen the tumour was pronounced not to be a sarcoma. If this represents the state of our knowledge here in London how can we expect that a hard-worked practitioner in the country can always give a correct certificate in a doubtful case?

Then when we come to the question of heredity and a patient says, "It is very strange; there was never anything of the kind in my family," how does he or she know that it is not in the family? People try to hide cancer, and it is this feeling of secrecy which helps to bring patients to us too late. It must have occurred to many practitioners to meet with cases of cancer where, for one reason or another, an operation has not been advisable and where the patient has died from something else. In such a case the friends may know nothing about it, and will truthfully say that so far as they know the disease is not "in the family."

The answer to the question, Is cancer of the breast curable by operation? is, in my opinion, most certainly in the affirmative; but not every case, even when it is apparently favourable in itself for operation. One life insurance office quite recently did not recognise as a cure a case where there was no return after seven years. The first thing to be decided, if possible, is what is a cure. A reasonable definition appears to be that the patient dies from some other disease than cancer, there being no sign of return. Within the scope of this definition a cure might be claimed if a patient died from any acute disease a few weeks after operation. This would be a true but not a legitimate claim, and it shows the difficulty of defining what is a cure, under the circumstances. The question of time, therefore, comes in, but it does not come in satisfactorily; because if a patient died 20 or 25 years after a cancerous breast had been removed no one could say positively that the disease might not have returned if she had lived another year. There must always be this doubt, and there may therefore be those who honestly hold that a cure cannot be obtained by operation. A space of three years has been arbitrarily fixed by some as the time after which a cure may be claimed. This is undoubtedly too short a time, but even suppose one fixed it at five years, there would still be some, though much less, doubt. Every year increases the patient's safety, and at the same time increases the difficulty of keeping track of the cases. The only positive gain in fixing a certain length of time as a cure is that it will cut out the patients who die from some other disease before the time limit is reached. There is another way of looking at this question of cure, and that is, can a cancerous growth which appears after, say, five or six years be reasonably called a recurrence of the original growth, or is it not a disease originating de novo? But this 
brings us back to the heredity and predisposition question. Speculations and theories of this kind do not do anything for the patient, and while I consider that many patients can be cured, there will be, with our present knowledge, always some doubt in the patient's mind after operation. A time limit may be sufficient for statistics, but it is not long enough for the patient.

Within the past 10 years I have seen four cases where operations had been performed on the mamma for malignant disease and where the whole of the gland had not been removed. To be quite correct it would be better to say that the patients had been told that the disease was cancer. In three of these four cases there was a return, and in the fourth there has not been, so far, any return, though I was consulted as to the propriety of a third operation, which had been proposed. As it is now five years since the second operation, performed 10 weeks after the first and more than three since I first saw the case, and as the patient has still two considerable pieces of breast, which I was informed swelled up considerably after a confinement, it is probable that the disease was of a benign nature. This, however, does not affect the statement that four cases have been seen in 10 years where apparently no effort had been made to remove the whole of the mammary gland when operated on for cancer.

It might be supposed that these operations had been performed by surgeons of little experience, but this was not the case. In one the operator was a surgeon to a general hospital in a large provincial town, and in two the operations were performed by two hospital surgeons in london. In the fourth case my father was consulted, and I do not know who the operator was. This one I simply saw as a curiosity, my father never having met with anything of the kind before. This may be a unique experience, and it may be rash to argue from such a small foundation, but when we bear so much of the very formidable operations advocated at present and see the patients come as they do, when the operation has failed to cure, with the evidence of an enormous scar to show how extensive the operation has been, are we not justified in thinking that there may be something not quite right? Have the operations in the past been performed as well as they might? and - what everyone knows but what seems to be kept in the background-is it not that there are many varieties of malignancy in what appear to be ordinary cases of cancer of the breast? To take the second question first, it may be, and at least ought to be, flogging a dead horse. Compare the case of a florid, clear-skinned young woman, 35 years of age, with a hard mass in the breast, which has been noticed for a month or six weeks and which is increasing in size, with that of a thin old woman 75 years of age, who has noticed a lump for some time, she does not exactly remember for bow long. Microscopically the structure of these two wrowths may be very much the same, but in the first the patient will be dead in a year after the operation, no matter how extensive it be ; and in the second five or even ten years may show bat little increase in the size of the growth. Nor is it only in the very old that we sometimes see comparatively slow growth of a scirrbous tumour, though the answer to this may be that all slow-growing tumours have been originally simple. This answer hardly holds for a case such as the following. A patient, 48 years of acre, consulted me nearly six years ago on account of a hard mass in the left breast situated at the upper and inner part. The history of the tumour was that it had been known of for about six years, as a medical man had been consulted at that time. My diagnosis of scirrhous cancer was corroborated by the late Sir James Paget, and also after the remoral of the breast. Rather more than a year ago there had been no return. As there had not been any perceptible increase in size in six years it is at least very improbable that the tumour was cancerous from the beginning. A case like this demonstrates also the difficulty of a time linist. Three years would undoubtedly be too short, when the tumour had been growing for at least double that length of time.

The average duration of life after a cancer of the breast has been discovered has been variously estimated at from abont three to four years. This means, of course, that a certain number would die before the average time was reached, or, in other words, that the malignancy in these cases was greater than in the others, and that consequently the tendency to return would be greater in them.

To come back to the question of the possibility of some of the operations in the past having been performed without sufficient care, we must clear up the question of return of the disease. A local return--that is to say, one close to where the disease had been removed or in direct communication with the surrounding lymphatics-must be differentiated from the reappearance of cancer in some other part of the body. The reason for the return in the first is, or at least may be, that the disease has not been removed with sufficient care or extensively enough. When the disease reappears in some other part of the body no blame can possibly be attached to the way in which the operation has been performed. An example of the first was a lady, 45 years of age, whose breast I removed for a scirrhous growth, the presence of which the patient had known of for at least two years. Barely 12 months afterwards a small growth of about the size of a pea was noticed under the skin and some distance from the scar. It was supposed to have come as the result of a kick while playing with a child. I removed it frcely, and also another at the end of the second year, which also was put down to a blow. Since this third operation the three-years limit has been passed. An example of the second was a case where the breast was removed and within five months there was deposit in the lungs, everything else being healthy. In the first there was a local recurrence because I had evidently not removed as much apparently healthy tissue as was necessary; in the second there was surely a centre of disease unconnected with the breast. Had one or both pectorals been removed in the first case the disease would have returned, because the deposit was in the fat superficial to the edge of the breast, and a similar operation would not have prevented the appearance of the disease in the lungs in the second case.

The Halsted operation is one which must have, from its sererity, a certain amount of mortality in weak subjects, while the removal of the breast and axillary glands is an operation unaccompanied by risk to life, except the accidental risk common to all operations, no matter how tricial. What is more necessary than the removal of one or both pectoral muscles is the careful removal of the fat superficial to the breast. It may be different in hospitals now, but 20 years ago it was common to see a fairly large piece of skin excised, and little or no precaution taken to remove the fat superficial to the remainder of the breast and so, when the wound was drawn together, a thick layer would often be seen to have been left behind. Comparatively recently it has been shown that the lymphatics do pass freely into the superficial fat, and it may be that if extra attention is paid to this part during the course of the extensive operations practised by some, the improved results are due to this rather than to the free remoral of muscle. To come back to the starting place, if a certain number of cases are operated upon where part of the gland only is removed, is it not possible that many more may not have sufficient tissue surrounding the breast removed and that it is in this way that the supposed necessity for the much enlarged and consequently more dangerous operation has come about?

Charles-street, $W$.

\section{Clintial 算otes:}

\section{MEDICAL, SURGICAL, OBSTETRICAL, AND} THERAPEUTICAL.

\section{NOTE ON AN UNUSUAL CASE OF SOARLET} FEVER.

By A. J. Rice OXLeY, M.D. DUb., M.R.C.S. ENG.

THE following case is of so unusual a character that it appears to me to be worthy of record.

On June 20th I was sent for to see a boy who was suffering from rash, sore-throat, and fever. The case was undoubtedly one of scarlet fever and was at once isolated. On the 26th free peeling commenced, the little patient meantime having done very well in every way. On the 30th he was allowed to sit up. On July 2nd the cervical glands, both sides, commenced to swell and become tender, although the temperature remained normal and the patient was in the best possible spirits. There was no albumin. He was, however, ordered to resume the fluid diet and was carefully kept in bed. 